\title{
Whole Sporozoite Vaccination: Duration between Successive Immunization Dictates the Fate of Protective Immunity \\ Rajesh Parmar ${ }^{1}$, Hardik Patel${ }^{2}$, Sarat K. Dalai ${ }^{1 *}$ \\ 'Institute of Science, Nirma University, Ahmedabad, Gujarat, India \\ ${ }^{2}$ Center for Global Infectious Disease Research, Seattle Children's Research Institute, Seattle, Washington
}

\section{Article Info}

\section{Article Notes}

Received: May 4, 2019

Accepted: May 31, 2019

\section{*Correspondence:}

Dr. Sarat Kumar Dalai, Institute of Science, Nirma University, Sarkhej-Gandhinagar Highway, Ahmedabad 382481, Gujarat, India; Email: sarat.dalai@nirmauni.ac.in.

C 2019 Dalai SK. This article is distributed under the terms of the Creative Commons Attribution 4.0 International License.

\section{Abstract}

Whole sporozoite vaccination (WSV) is considered a gold standard for inducing and providing sterile protection against Plasmodium infection. Multiple doses of immunizations with radiation-attenuated sporozoites (RAS) is essential for establishing complete sterile protection against Plasmodium infection in mice as well as in humans. In our recently published article ${ }^{1}$, we have shown that the pattern of vaccination with RAS determines the degrees of protection in mice and frequent immunization with RAS in optimum time duration helps in generating minimum threshold liver-stage (LS) specific CD8 ${ }^{+} \mathrm{T}$ cell memory responses leading to sterile protection. Further, we have shown that the alterations in successive RAS immunization could possibly affect the induction of sterile protection. In summary, animals receiving four successive doses generated $100 \%$ sterile protection. However, three successive doses with the same parasite inoculum as four doses, could induce sterile protection in $\sim 50 \%$ mice. Interestingly, mice immunized with the same 3 doses, but with longer gap, could not survive the challenge.

\section{Introduction}

Despite tremendous development of healthcare system, malaria in the $21^{\text {st }}$ century still remains a serious challenge to human health particularly to children under 5 years of age. Malaria caused by protozoan parasites of Plasmodium genus, remains a major health problem worldwide with almost $35 \%$ of the human population is at risk of becoming infected ${ }^{2}$. This mosquito-borne disease is mainly responsible for illness and death among infants and young children, particularly in Africa and southeast Asia. The pregnant women are also highly susceptible to malaria infection; upon infection they develop anemia, prematurely deliver baby with low birthweight, often causing death of infants. Further, children who survived the malaria infection suffer from various complications including mental and physical health.

The Plasmodium parasite has a complex life cycle ${ }^{3}$ and is adaptable to various environmental conditions (Figure 1). Development of all Plasmodium species occurs in two different hosts: the definitive host is the mosquito, where the parasite undergoes sexual reproduction, while human is the intermediate host for Plasmodium falciparum, wherein the asexual multiplication occurs. The life cycle of Plasmodium is broadly divided into three phases: asymptomatic pre-erythrocytic (PE) stage (approx. 5-6 days in humans or 2-3 days in rodents), which initiates the infection in the liver; the asexual erythrocytic stage, which causes disease symptoms; and the gametocyte stage, that includes the infection of the mosquitoes. 


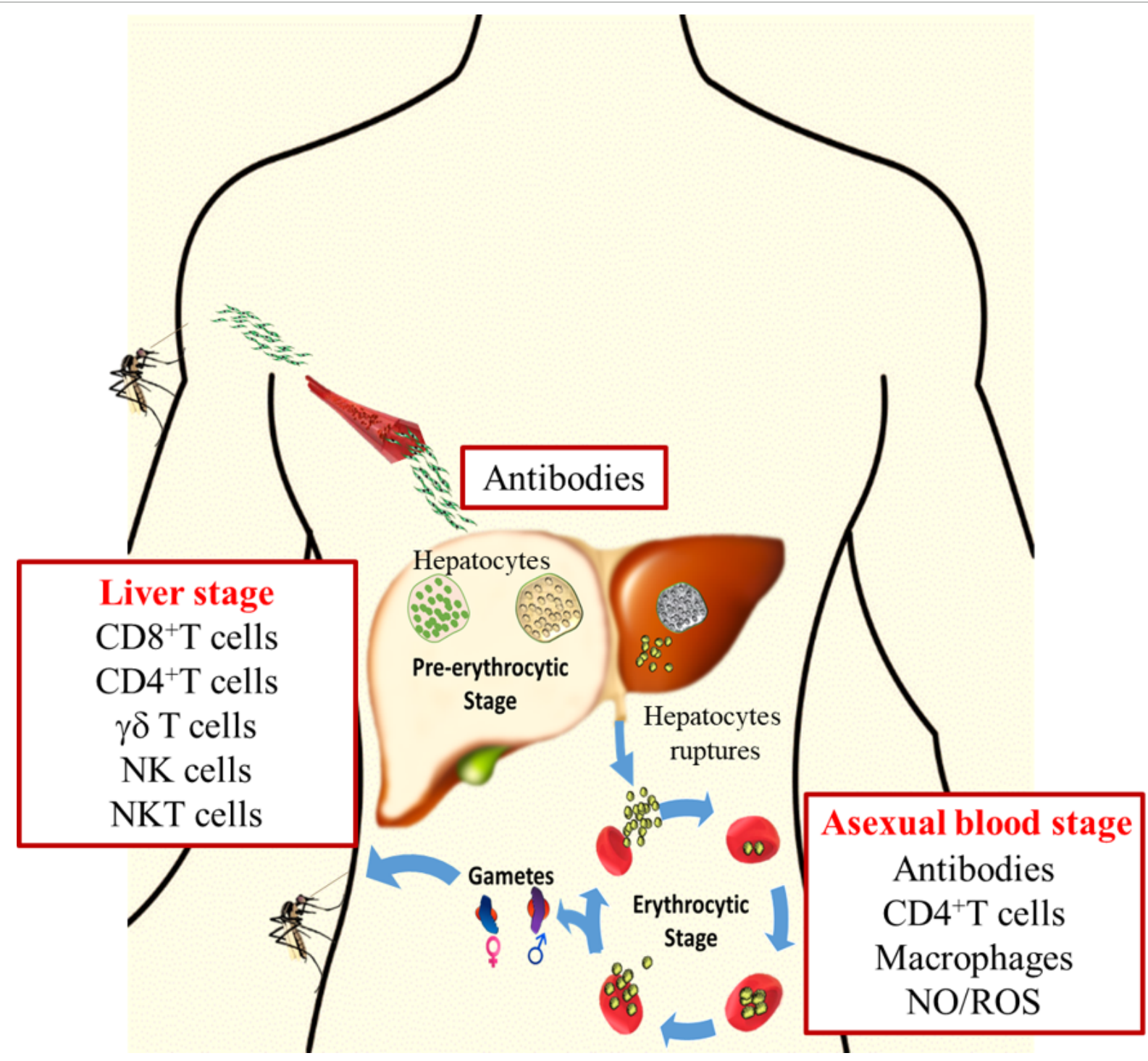

Figure 1 Life cycle of malaria parasite and possible immune mechanisms at various stages of the Plasmodium life cycle in the mammalian host. Infected female Anopheles mosquito delivered the sporozoites at the time of blood meal into the bloodstream. They pass quickly in the human liver where they multiply asexually as merozoites over the next 7-10 days, causing no symptoms. Parasitic stage in the liver is clinically silent. Fever and severe malaria are associated with the parasite cycle in the blood, as well as adherence of infected RBCs to the blood vessel endothelium and to each other. Then merozoites invade erythrocytes and multiply until the cells rupture, releasing merozoites and infect mature erythrocytes. This cycle is repeated, causing fever each time the parasite breaks free. Some proportion of the released merozoites develop into gametocyte that subsequently infect another mosquito. In the liver stage, protective immune response is generated by $\mathrm{CD}^{+} \mathrm{T}$ cells and cytokine. At the blood stage, antibodies and $\mathrm{CD} 4^{+} \mathrm{T}$ cells are mainly responsible for the controlling the Plasmodium infection.

The PE stage begins when an infected female Anopheles mosquito delivers a small number of infectious sporozoites into the bloodstream while biting the host for blood meal, these sporozoites rapidly travel to the liver infect the hepatocytes. Before infecting the hepatocytes, the sporozoites are supposed to pass through a number of hepatocytes. In the liver, a single sporozoite gives rise to thousands of asexual parasites called merozoites that rupture the hepatocytes and end up in the blood stream. Each sporozoite forms a schizont containing 10,000-
40,000 merozoites ${ }^{4}$. Further, the released merozoites infect red blood cells (RBCs) and multiply within them until the RBCs burst open releasing the merozoites. This cycle is repeated, causing clinical symptoms of malaria, each time the parasite breaks free. Subsequently, the gametocyte stage begins in the female Anopheles mosquitos with the gametocytes undergoing fertilization and maturation to form the ookinetes that further develop to the oocyst in which sporozoites are formed. After maturation, the oocysts burst and release the sporozoites that migrate to 
the salivary glands of the mosquito and get ready for the next transmission into the mammalian host.

\section{Effectiveness of Anti-Malarial Drug, Emergence Drug Resistance, Need of the Vaccine}

Use of various anti-malarial drugs, including artemisinin-based combinational therapy and vector control measures e.g. insecticide-treated nets have resulted in mixed outcome ${ }^{5,6}$. In certain regions of Africa, while these interventions have been linked with a temporary 50\% reduction in incidence, other regions of Africa and some parts of the world, such as, Amazonia, have noticed the increase in the incidence of malaria ${ }^{7,8}$. Most importantly, $P$. falciparum has been shown to be acquiring and rapidly spreading resistance to anti-malarial drugs ${ }^{9,10}$. At present we have to deal with multi-drug-resistant parasites against most potent drug, including chloroquine and artemisinin, and the emergence of insecticide-resistant mosquitoes making it very difficult to control malaria. Given the enormous genetic plasticity of the parasite, the emergence of antimalarial drug resistance is inevitable and thus a major concern ${ }^{3}$. Therefore, it is critical to have an effective vaccine to control or possibly eradicate malaria.

Attempts have been made to develop malaria vaccine; morethan 10 P.falciparum malariavaccinesateitheradvanced preclinical or clinical stages of evaluation are designed to induce protective antibody-or cell-mediated immune responses against the liver or blood-stage infection. The vaccines include, $\mathrm{PE}$ vaccines that target the asymptomatic phase and aim either to prevent the sporozoites from getting into the hepatocytes or to destroy infected hepatocytes e.g., RAS vaccine, GAP (Genetically attenuated parasite) vaccines, RTS,S/AS01, and CVac $^{11}$. Further, erythrocytic vaccines targeting the symptomatic stage aim to stop the rapid invasion and asexual reproduction of the parasite in the RBCs e.g., chemically attenuated parasites, AMA1-RON2, and PfRH5 ${ }^{11}$. Lastly, the transmission blocking vaccine that targets to kill the vector, the Anopheles mosquito, to stop further spread of the parasite e.g. Pfs25, Pfs230, and Pfs4711.

Only one malaria vaccine candidate i.e., RTS,S/AS01 has approval for use in countries where malaria is endemic and a small number of volunteers are shown to be protected with low efficacy ${ }^{12}$. Further, malaria vaccine development is hindered due to the complexity of the parasite and its life cycle $^{13}$, extensive antigenic variation ${ }^{14}$, and a poor understanding of the interaction between P. falciparum and the human immune system ${ }^{15}$.

\section{Natural Immunity against Malaria}

Various studies have suggested that protective immunity can be achieved naturally among young children as well as adults, who have lived in an malaria endemic area for 6-7 years due to repeated exposure of malarial parasites ${ }^{15,16}$.
However, the natural immunity is vanishes when immune adults leave the malaria endemic settings, suggesting the fact that repeated exposure to malarial parasite is critical to induce and maintain the immunity. Generation of natural immunity in response to severe disease is shown to be developed among the children living in malaria endemic areas where transmission is high; on the contrary, the same is not true for the children living in areas of low transmission suggesting that frequent exposure to malaria parasite is required to generate and maintain immunity. Furthermore, natural immunity is observed as the collective process of continuous exposure to infectious malaria parasite over the years, which yields a sufficiently diverse repertoire of immune responses.

Individuals repeatedly exposed naturally to Plasmodium parasites develop antibodies against the PE as well as erythrocytic stage, which act on sporozoite, liver-stage, and various blood-stage malaria parasites ${ }^{16}$. Additionally, effector mechanism of naturally acquired immunity include the array of cytokines especially interferon-gamma (IFN$-\gamma$ released that act against all stages of the Plasmodium infection ${ }^{17,18}$. During natural infection in humans, cytotoxic $\mathrm{CD}^{+} \mathrm{T}$ cells specific for certain Plasmodium antigens detected in many individuals with previous contact with P. falciparum ${ }^{19}$ are thought to provide protection. Further, Plasmodium antigen specific $\mathrm{CD}^{+}{ }^{+} \mathrm{T}$ cells are shown to activate macrophages that phagocytose and eliminate intra-erythrocytic parasites and free merozoites ${ }^{20}$.

Importance of Vaccine Targeting Liver-Stage against Plasmodium

Liver-stage (LS) in the Plasmodium life cycle is clinically silent stage and the number of infected cells is very low. Hence, it is an impeccable target for vaccine-induced protective immune responses. Since, this stage lasts for about 6-7 days in humans, and potential whole-sporozoite anti-malarial vaccines can confine the parasite in the liver and preventing parasite from further development into infectious blood-stages infections ${ }^{21}$.

A vaccine that would efficiently target the infected hepatocytes would prevent both the clinical symptoms of the disease and the infection ${ }^{22}$. Remarkable efforts are being made for the development of an effective vaccine, but only RTS, S/AS01, (Mosquirix) a pre-erythrocytic subunit vaccine launched recently in the market has shown to induce antibody and $\mathrm{CD}^{+} \mathrm{T}$ cell response against sporozoite-expressed surface protein i.e., circum sporozoite protein $(\mathrm{CSP})^{23-28}$. The main target group of Mosquirix for active immunization are children aged between 6 weeks to 17 months against malaria caused by P. falciparum. Although the efficacy is relatively good in terms of protective immunity, the protection generated by RTS,S/AS01 is very short-lived and last up to 6 months ${ }^{23}$. 


\section{Liver-stage Specific $\mathrm{CD8}^{+} \mathrm{T}$ Cell Response against Plasmodium Infection}

Whole sporozoite vaccination (WSV) has demonstrated relatively high vaccine efficacy, but depends on repeated frequent doses of WSV to ensure sterile immunity in humans as well as rodents ${ }^{8,29-31}$. WSV includes RAS, GAP, and CPS (infectious sporozoites under chloroquine cover $^{32}$. In mice immunized with RAS, the critical role of $\mathrm{CD}^{+} \mathrm{T}$ cells in generating sterile protection was first established in the $1980 \mathrm{~s}^{33}$. Various experimental data including ours suggest that the WSV would induce multifunctional long-lived memory $\mathrm{CD}^{+} \mathrm{T}$ cells against infected hepatocytes ${ }^{1-38}$. Protective immunity is lost with the attrition of LS CD8 ${ }^{+} \mathrm{T}$ cells (Figure 2). For achieving the complete sterile protection, a minimum threshold LS $\mathrm{CD}^{+} \mathrm{T}$ cell is required ${ }^{1}$. Reports from various investigators, including ours, have shown a requirement of the presence of a minimal threshold (provide the $\%$ of multifunctional $\mathrm{CD}^{+} \mathrm{T}$ cells) of activated memory $\mathrm{CD}^{+} \mathrm{T}$ cells in liver for sterile protective immunity against malaria ${ }^{1}$. LS specific effector $\mathrm{CD}^{+} \mathrm{T}$ cells are producers of a large quantity of IFN- $\gamma$, perforin and granzyme and highly potent to eliminate the infected hepatocytes ${ }^{1,35}$.

\section{Gap between Successive Immunizations with RAS} Induced Generation of $\mathrm{CD}^{+} \mathrm{T}$ cells

Various studies, including ours, have shown that multiple doses of RAS given in biweekly interval leads to induction of sterile protection ${ }^{1,29,34,35}$. In our recent study, however, we have shown that the longer the duration (i.e., $>6$ weeks) between two successive immunizations with RAS of Plasmodium berghei fails to provide sterile protection ${ }^{1}$. In addition, we have demonstrated that that varying degree of protection found in mice inoculated with RAS correlates with the presence of IFN- $\gamma$ producing $\mathrm{CD}^{+} \mathrm{T}$ effector memory $\left(\mathrm{T}_{\mathrm{EM}}\right)$ cells in the liver ${ }^{1}$. Our study emphasizes the requirement of frequent inoculations of RAS in the optimum interval between successive immunization to induce sterile protection and irregular immunization would lead to failure in achieving the protective immunity and the minimal threshold of IFN- $\gamma$ producing CD8 ${ }^{+} \mathrm{T}_{\mathrm{EM}}$ cells in the liver required during malaria infection to ensue protection.

We found that the presence of IFN- $\gamma$ or multifunctional $\mathrm{CD}^{+} \mathrm{T}_{\mathrm{EM}}$ cells in the liver is influenced by the nature of inoculation (i.e. frequency of exposure) which further reflects in the degree of protection. The findings provide the clue that the cumulative parasite antigen load during vaccination might not be the sole determining factor, rather it depends upon the frequency of immunization. By looking at the IFN- $\gamma$ producing $\mathrm{CD}^{+} \mathrm{T}$ cells pre- and post-challenge we demonstrate the potential of endogenous memory $\mathrm{CD}^{+} \mathrm{T}$ cells, presumably specific to multiple antigens, to play an important role in providing sterile protection after

\section{A. Pre-existing $\mathrm{CD8}^{+} \mathrm{T}_{\mathrm{EM}}$ cells in liver}
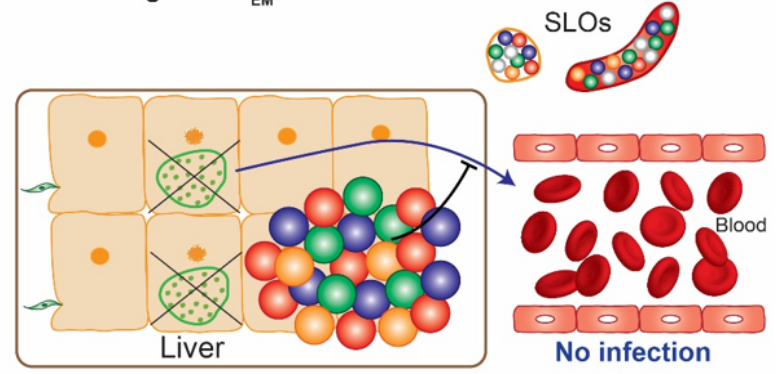

No infection

B. Recruitment of reactivated $\mathrm{CD}^{+} \mathrm{T}_{\mathrm{EM}}$ cells from SLOs to meet the required threshold

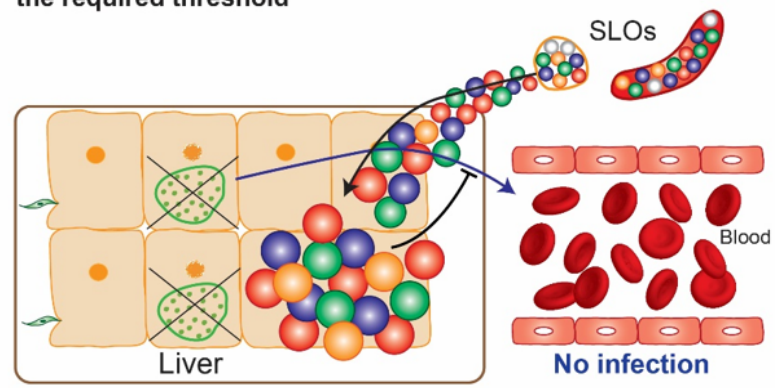

C. Failure of meeting the required threshold of $\mathrm{CD}^{+} \mathrm{T}_{\mathrm{EM}}$ cells in liver

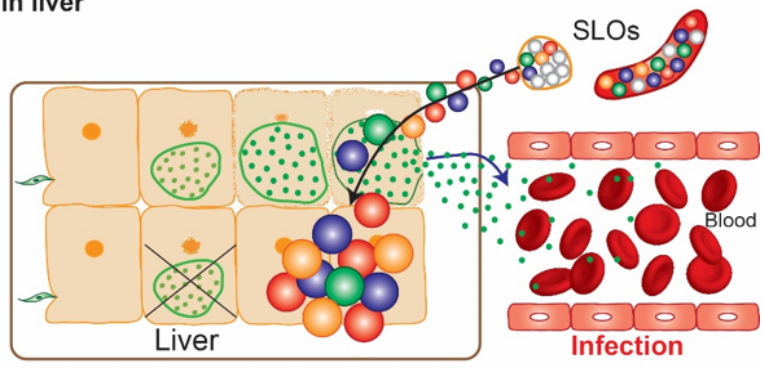

Figure 2. Schematics of minimal threshold $\mathrm{CD8}^{+} \mathrm{T}_{\mathrm{Eм}}$ cells attained/accumulated in the liver of immune host ensuring protection. Plasmodium liver-stage specific $\mathrm{CD}^{+} \mathrm{T}_{\mathrm{EM}}$ cells are required to be present in the liver in order to achieve sterile protection (A) If there are adequate numbers of effector memory $\mathrm{T}$ cells present in the liver before sporozoites invade the hepatocytes, the host might be protected as effector $\mathrm{T}$ cells would fight and eliminate the infected hepatocytes. (B) If the number of effector memory $\mathrm{T}$ cells present in the liver is not enough to provide protection but memory cells residing in the nearby secondary lymphoid organs get reactivated and join the pool of liver-resident $\mathrm{T}$ cells making up the desired frequencies, the host could be rescued from being infected. (C) If the liverresident $\mathrm{T}$ cell numbers are low and the memory $\mathrm{T}$ cells residing in the secondary lymphoid organs are also low and/or fail to get reactivated, the overall $\mathrm{T}$ cells in the liver would be below the frequency of cells required to fight the infection, the host becomes susceptible to challenge.

attaining the desired frequencies (Fig. 2). From this study, we speculate that sterile immunity might be retained, if the frequencies of IFN- $\gamma$ or multifunctional $\mathrm{CD}^{+} \mathrm{T}_{\mathrm{EM}}$ cells are either maintained until the subsequent infection or attained while the parasite is developing in the liver during the challenge. The basic information generated by this 
study may aid in rationalizing the whole sporozoite vaccine regimen for inducing sterile protection.

\section{Conclusions}

It will not be feasible to eradicate malaria through vaccination unless we understand the complexity of the induction and generation of sterile protection in response to RAS. Mass vaccination has its own challenges so far as compliance is concerned. Our study ${ }^{1}$ highlights some of the unanswered questions regarding the generation of sterile immunity to Plasmodium, the tools needed to address these questions, and the research that is underway. In addition, understanding malaria immunity in the context of developing a truly universal malaria vaccine is the focus of a new strategic plan for the developing next generation malaria vaccine research. Until this is achieved, we will continue to chase and fail to catch the constantly moving target of Plasmodium. Our results as well as others emphasize the requirement of frequent inoculations of RAS, to induce sterile protection, in stipulated time between two successive immunizations. The presence of required threshold of IFN- $\gamma$ or multifunctional $\mathrm{CD}^{+} \mathrm{T}_{\mathrm{EM}}$ cells in the liver is influenced by the nature of inoculation (i.e. frequency of exposure) leading to generation of different degree of protection.

\section{Abbreviations}

RAS (Radiation attenuated sporozoites), GAP (Genetically attenuated parasite), IFN (Interferon), T effector memory $\left(\mathrm{T}_{\mathrm{EM}}\right)$

\section{Contributors Statement Page}

Dr. Parmar drafted the manuscript and prepared the figures, Dr. Dalai edited the manuscript, Dr. Hardik Patel assisted in editing and preparing the figures.

\section{Acknowledgement}

The authors thank all the present members of the laboratory for their contributions to the work described herein. Thanks to DST (EMR/2014/000535), DBT (BT/ PR3130/MED/12/519/2011), Govt. of India, New Delhi; and GSBTM (GSBTM/MD/ PROJECTS/SSA/2243), GUJCOST (GUJCOST/MRP/2014-15/1629), Govt. of Gujarat and Nirma University for financial support to SKD lab.

\section{References}

1. Patel H, Yadav N, Parmar R, et al. Frequent inoculations with radiation attenuated sporozoite is essential for inducing sterile protection that correlates with a threshold level of Plasmodia liver-stage specific CD8+ T cells. Cell Immunol. 2017; 317: 48-54.

2. WHO. WHO Malaria Report: World Health Organization; 2018.

3. Miller LH, Ackerman HC, Su XZ, et al. Malaria biology and disease pathogenesis: insights for new treatments. Nat Med. 2013; 19: 156167.

4. Aly AS, Vaughan AM, Kappe SH. Malaria Parasite Development in the
Mosquito and Infection of the Mammalian Host. Annual review of microbiology. 2009; 63: 195-221.

5. Bhattarai A, Ali AS, Kachur SP, et al. Impact of artemisinin-based combination therapy and insecticide-treated nets on malaria burden in Zanzibar. PLoS Med. 2007; 4: e309-e309.

6. Eastman RT, Fidock DA. Artemisinin-based combination therapies: a vital tool in efforts to eliminate malaria. Nature reviews Microbiology. 2009; 7: 864-874.

7. Jongo SA, Shekalaghe SA, Church LWP, et al. Safety, Immunogenicity, and Protective Efficacy against Controlled Human Malaria Infection of Plasmodium falciparum Sporozoite Vaccine in Tanzanian Adults. Am J Trop Med Hyg. 2018; 99: 338-349.

8. Sissoko MS, Healy SA, Katile A, et al. Safety and efficacy of PfSPZ Vaccine against Plasmodium falciparum via direct venous inoculation in healthy malaria-exposed adults in Mali: a randomised, doubleblind phase 1 trial. The Lancet Infectious diseases. 2017; 17: 498-509.

9. Dondorp AM, Fanello CI, Hendriksen IC, et al. Artesunate versus quinine in the treatment of severe falciparum malaria in African children (AQUAMAT): an open-label, randomised trial. Lancet. 2010; 376: 1647-1657.

10. Dondorp AM, Yeung S, White L, et al. Artemisinin resistance: current status and scenarios for containment. Nature reviews. Microbiology. 2010; 8: 272-280.

11. Tuju J, Kamuyu G, Murungi LM, et al. Vaccine candidate discovery for the next generation of malaria vaccines. Immunology. 2017; 152: 195206.

12. Hill AV, Reyes-Sandoval A, O'Hara G, et al. Prime-boost vectored malaria vaccines: progress and prospects. Human vaccines. 2010; 6: 78-83.

13. Gardner MJ, Hall N, Fung E, et al. Genome sequence of the human malaria parasite Plasmodium falciparum. Nature. 2002; 419: 498511.

14. Scherf A, Lopez-Rubio JJ, Riviere L. Antigenic variation in Plasmodium falciparum. Annual review of microbiology. 2008; 62: 445-470.

15. Langhorne J, Ndungu FM, Sponaas AM, et al. Immunity to malaria: more questions than answers. Nature immunology. 2008; 9: 725-732.

16. Doolan DL, Dobano C, Baird JK. Acquired immunity to malaria. Clinical microbiology reviews. 2009; 22: 13-36. Table of Contents.

17. Dodoo D, Hollingdale MR, Anum D, et al. Measuring naturally acquired immune responses to candidate malaria vaccine antigens in Ghanaian adults. Malaria journal. 2011; 10: 168.

18. Anum D, Kusi KA, Ganeshan $\mathrm{H}$, et al. Measuring naturally acquired ex vivo IFN-gamma responses to Plasmodium falciparum cell-traversal protein for ookinetes and sporozoites (CelTOS) in Ghanaian adults. Malaria journal. 2015; 14: 20.

19. Plebanski M, Aidoo M, Whittle HC, et al. Precursor frequency analysis of cytotoxic T lymphocytes to pre-erythrocytic antigens of Plasmodium falciparum in West Africa. J Immunol. 1997; 158: 2849-2855.

20. Stevenson MM, Ing R, Berretta F, et al. Regulating the adaptive immune response to blood-stage malaria: role of dendritic cells and $\mathrm{CD}^{+}{ }^{+}$Foxp $^{+}$regulatory T cells. Int J Biol Sci. 2011; 7: 1311-1322.

21. Holz LE, Fernandez-Ruiz D, Heath WR. Protective immunity to liverstage malaria. Clinical \& Translational Immunology. 2016; 5: e105.

22. Bertolino P, Bowen DG. Malaria and the liver: immunological hideand-seek or subversion of immunity from within? Frontiers in microbiology. 2015; 6: 41-41.

23. RTS,S Clinical Trials Partnership, Agnandji ST, Lell B, et al. A phase 3 trial of RTS,S/AS01 malaria vaccine in African infants. The New England journal of medicine. 2012; 367: 2284-2295. 
24. Moncunill G, De Rosa SC, Ayestaran A, et al. RTS,S/AS01E Malaria Vaccine Induces Memory and Polyfunctional T Cell Responses in a Pediatric African Phase III Trial. Front Immunol. 2017; 8: 1008.

25. Agnandji ST, Lell B, Soulanoudjingar SS, et al. First results of phase 3 trial of RTS,S/AS01 malaria vaccine in African children. The New England journal of medicine. 2011; 365: 1863-1875.

26. Bejon P, White MT, Olotu A, et al. Efficacy of RTS,S malaria vaccines: individual-participant pooled analysis of phase 2 data. The Lancet Infectious Diseases. 2013; 13: 319-327.

27. Kester KE, Cummings JF, Ofori-Anyinam O, et al. Randomized, double-blind, phase 2a trial of falciparum malaria vaccines RTS,S/ AS01B and RTS,S/AS02A in malaria-naive adults: safety, efficacy, and immunologic associates of protection. The Journal of infectious diseases. 2009; 200: 337-346.

28. Stoute JA, Slaoui M, Heppner DG, et al. A preliminary evaluation of a recombinant circumsporozoite protein vaccine against Plasmodium falciparum malaria. RTS,S Malaria Vaccine Evaluation Group. The New England journal of medicine. 1997; 336: 86-91.

29. Epstein JE, Paolino KM, Richie TL, et al. Protection against Plasmodium falciparum malaria by PfSPZ Vaccine. JCI Insight. 2017; 2: e89154.

30. Ishizuka AS, Lyke KE, DeZure A, et al. Protection against malaria at 1 year and immune correlates following PfSPZ vaccination. Nat Med. 2016; 22: 614-623.

31. Lyke KE, Ishizuka AS. Attenuated PfSPZ Vaccine induces straintranscending $\mathrm{T}$ cells and durable protection against heterologous controlled human malaria infection. 2017; 114: 2711-2716.
32. Bijker EM, Bastiaens GJ, Teirlinck AC, et al. Protection against malaria after immunization by chloroquine prophylaxis and sporozoites is mediated by preerythrocytic immunity. Proceedings of the National Academy of Sciences of the United States of America. 2013; 110: 7862-7867.

33. Nussenzweig RS, Vanderberg J, Most H, et al. Protective immunity produced by the injection of x-irradiated sporozoites of plasmodium berghei. Nature. 1967; 216: 160-162.

34. Roestenberg M, McCall M, Hopman J, et al. Protection against a malaria challenge by sporozoite inoculation. The New England journal of medicine. 2009; 361: 468-477.

35. Parmar R, Patel H, Yadav N, et al. Infectious Sporozoites of Plasmodium berghei Effectively Activate Liver CD8 $\alpha+$ Dendritic Cells. Frontiers in Immunology. 2018; 9.

36. Schmidt NW, Butler NS, Badovinac VP, et al. Extreme CD8 T Cell Requirements for Anti-Malarial Liver-Stage Immunity following Immunization with Radiation Attenuated Sporozoites. PLoS Pathogens. 2010; 6: e1000998.

37. Spencer AJ, Longley RJ. The Threshold of Protection from Liver-Stage Malaria Relies on a Fine Balance between the Number of Infected Hepatocytes and Effector CD8+ T Cells Present in the Liver. 2017; 198: 2006-2016.

38. Van Braeckel-Budimir N, Harty JT. CD8 T-cell-mediated protection against liver-stage malaria: lessons from a mouse model. Frontiers in Microbiology. 2014; 5: 272. 